tactile sensitivity on the SSP, were highly correlated with measures of psychopathology on the $\mathrm{CBCL}$, including aggressive and delinquent behavior and concerns about body and health. The authors propose two distinct sensory subgroups within $\mathrm{ADHD}$, one with normal sensory responses and one with SMD. (Mangeot SD, Miller LJ, McIntosh DN et al. Sensory modulation dysfunction in children with attention-deficit-hyperactivity disorder. Dev Med Child Neurol June 2001;43:399-406). (Respond: Lucy Jane Miller PhD OTR, Department of Rehabilitation Medicine, University of Colorado Health Sciences Center Research Office, 1901 W Littleton Boulevard, Littleton, CO 80120).

COMMENT. Sensory modulation is defined as the ability to regulate the degree and intensity of responses to sensory input, to optimize the performance and adaptation to the environment. Sensory modulation dysfunction (SMD) may present as sensation seeking or sensation avoiding behaviors. Seeking sensation behaviors include touching others too often or too hard, overactive and risky behavior, and repeated tapping or banging. Avoiding sensation behaviors are aggressive response or withdrawal from touch, fear of playground and car rides, over response to hugging and dislike for sports. Problems with sensitivity to stimuli such as touch may lead to emotional and behavior problems including aggressive responses. Treatments aimed at remediation of SMD among children with $\mathrm{ADHD}$ may prevent the development of comorbid oppositional defiance disorder.

\title{
CORTICAL LOCALIZATION OF READING IN NORMAL CHILDREN
}

Brain regions involved in processing written text while reading were identified by fMRI in 9 right-handed normal children, ages 7.9 to 13.3 years. While reading Aesop's Fables and responding to a reading response naming test, the fMRI showed strong activation in the left middle temporal gyrus and left midfrontal gyrus and variable activation in left inferior frontal gyrus. Reading Aesop's Fables activated twice as many pixels in temporal cortex as the Read Response Naming task. Activation in the right middle temporal region was small. Reading text paradigms and fMRI may be useful in determining language dominance in children with epilepsy before surgery. (Gaillard WD, Pugliese M, Grandin $\mathrm{CB}$ et al. Cortical localization of reading in normal children. An fMRI language study. Neurology July (1 of 2) 2001;57:47-54). (Reprints: Dr WD Gaillard, Department of Neurology, Children's National Medical Center, 111 Michigan Ave NW, Washington, DC 20010).

COMMENT. Reading in normal children is lateralized and localized to specific brain areas by middle to late childhood. Strong laterality in left temporal receptive and anterior language areas is identified in right handed subjects. Some but lesser activation is also present in the homologous non-dominant right temporal cortex.

\section{MUSCLE DISORDERS}

\section{ACUTE ONSET INFANTILE SPINAL MUSCULAR ATROPHY}

Two infants who were asymptomatic at birth and presented at 3 and 6 months of age with acute onset generalized muscle weakness and hypotonia following respiratory infection are reported from the Schneider's Children Hospital, New Hyde Park, NY. A suspected diagnosis of Guillain-Barre syndrome and corroborating nerve conduction studies led to a trial of immunoglobulin 
therapy, with minimal and questionable benefit. Subsequent DNA analyses on blood showed homozygous deletion mutation in exons 7 and 8 of the survival motor neuron $(\mathrm{SMN})$ gene. The final molecular diagnosis was SMA type I. One patient died at 5 months of age of pneumonia and respiratory failure, and the other was currently surviving on a respirator. (Ravid S, Topper L, Eviatar L. Acute onset of infantile spinal muscular atrophy. Pediatr Neurol May 2001;24:371-372). (Dr Eviatar, Division of Pediatric Neurology, Schneider's Children Hospital, New Hyde Park, NY 11040).

COMMENT. Several atypical presentations of infantile spinal muscular atrophy are cited from the literature. The acute onset of weakness and areflexia following a respiratory infection suggested a Guillain Barre syndrome, and the NCS were confirmatory. The very young age, less than 6 months, and the absent response to immunoglobulin were against this diagnosis. SMA type I was established by DNA molecular analyses. The final outcome with respiratory failure was expected.

\section{FACIAL ELECTROMYOGRAPHY IN NEONATAL FACIAL PALSY}

Electromyography (EMG) studies of 172 children with congenital facial palsy were analysed retrospectively at the Hopital d'Enfants Armand-Trousseau, Paris, France. The first clinical and EMG exams were done from 3 to 90 days after birth in 103 infants, 3 to 12 months in 49 , and from 1 to 2 years in 20 children. In 85 with unilateral congenital facial paralysis without malformation, 48 were attributed to perinatal forceps or other trauma. Clinical and EMG monitoring showed slow nerve regeneration and muscular reinervation even after 2 years. Asymmetrical crying facies occurred in 51 infants, the EMG showed partial denervation of the depressor anguli oris and depressor labia inferioris, and a diagnosis of partial facial palsy with only the lower lip involved. Orofacial malformations with asymmetrical facial motility were studied in 33 infants. EMG activity was either absent (10 infants), neurogenic (9), or myopathic (14). Pseudoparalysis asymmetry of postural origin occurred in 5 of 172 cases of congenital facial palsy. The EMG was normal and the functional asymmetry resolved within 2 months. Mobius syndrome in 22 infants had heterogeneous EMG findings with predominance of axonal loss. Congenital facial diplegia with muscular diseases, eg myotonic dystrophy, congenital muscular dystrophy, metabolic myopathies, facioscapulohumeral dystrophy with early onset facial weakness. In Orofacial apraxia with cerebral palsy, EMG of facial muscles is normal, except when brain stem nuclei are involved. In 12 infants with dysphagia at birth and failure to thrive, a neurogenic EMG indicated lesions of VII to XII cranial nerves. (Renault F. Facial electromyography in newborn and young infants with congenital facial weakness. Dev Med Child Neurol June 2001;43:421427). (Francis Renault MD, Unite de Neurophysiologie Clinique, Hopital d'Enfants ArmandTrousseau, 26 avenue du Docteur Arnold-Netter, 75571 Paris 12, France).

COMMENT. The author advocates EMG of facial muscles in infants to differentiate causes of congenital facial palsy and to evaluate course and prognosis. Electrodiagnostic studies of Mobius syndrome may shed light on the pathophysiology. The author provides details regarding his technique of EMG and findings, and values for motor latencies of facial muscles and facial nerve conduction velocities from birth $(19 \mathrm{~m} / \mathrm{s})$ to 3 years $(39 \mathrm{~m} / \mathrm{s})$. 